\title{
High precision two photon spectroscopy in hydrogen isotopes for the measurement of the electric field in plasmas generated in hollow cathode discharges
}

\section{Espectroscopía de dos fotones de alta precisión en isótopos de hidrógeno para la medida del campo eléctrico en plasmas generados en descargas de cátodo hueco}

\author{
V. Gonzalez-Fernandez $1,2, \mathrm{~S}^{*}$ \\ ${ }^{1}$ Dpto. de Física Teórica, Atómica y Óptica, Facultad de Ciencias, Universidad de Valladolid, Paseo de \\ Belén 7, CP 47011, Valladolid, España \\ ${ }^{2}$ Dpto. de Óptica, Universidad Complutense de Madrid, Avenida Arcos de Jalón s/n, CP 28037 Madrid, España \\ (*) E-mail: veronicagf@ucm.es \\ S: miembro de SEDOPTICA / SEDOPTICA member \\ Received: 08/10/2020 Accepted: 29/11/2020 \\ DOI: 10.7149/OPA.53.4.51050

\begin{abstract}
:
In this thesis, the intensity of the local electric field in the cathode region of a hollow cathode discharge operated in hydrogen was measured, in a wide range of pressures and currents to study the influence of the diameter (10 and $15 \mathrm{~mm}$ ) and material of the cathode used (stainless steel and tungsten). The method is based on the Stark effect of the level n=2, via the absorption of two $243 \mathrm{~nm}$ photons, circularly polarized in opposite directions, to eliminate the Doppler effect. When the atom is in the $2 \mathrm{~S}$ state, it photo-ionizes, modifying the impedance of the plasma, which can be detected as the voltage drop on a resistor (optogalvanic spectroscopy). The local electric field is determined by
\end{abstract} \\ comparing the separation of the Stark components with the theoretical values.
}

Key words: Spectroscopy, laser, plasma, hydrogen

\section{RESUMEN:}

En esta tesis se midió la intensidad del campo eléctrico local en la zona de cátodo en una descarga de cátodo hueco en régimen de glow-discharge operada en hidrógeno, en un amplio rango de presiones y corrientes para estudiar la influencia del diámetro $(10$ y $15 \mathrm{~mm})$ y material del cátodo empleado (acero inoxidable y tungsteno). El método se basa en el estudio del efecto Stark del nivel $n=2$, mediante la absorción de dos fotones de $243 \mathrm{~nm}$, circularmente polarizados en sentidos opuestos y en direcciones contrarias, para eliminar el efecto Doppler. Cuando el átomo se encuentra en el estado 2S, se fotoioniza modificando la impedancia del plasma, que puede ser detectada como la calda del voltaje en una resistencia (espectroscopia optogalvánica). El campo eléctrico local se determina comparando la separación de las componentes Stark con los valores teóricos.

Palabras clave: Espectroscopia, láser, plasma, hidrógeno

\section{REFERENCES AND LINKS / REFERENCIAS Y ENLACES}

[1] H. R. Griem, Principles of Plasma Spectroscopy. Cambridge University Press, (2005)

[2] K. Muraoka and M. Maeda. Laser-Aided Diagnostics of Plasmas and Gases. IoP Publishing, Bristol, (2001)

[3] R. W. Boyd, Nonlinear Optics. Academic Press (2003) 
[4] V. Gonzalez-Fernandez. Espectroscopia de dos fotones de alta precisión en isótopos de hidrógeno para la medida del campo eléctrico en plasmas generados en descargas de cátodo hueco. Tesis Doctoral. Universidad de Valladolid (2018)

[5] W. Lochte-Holtegreven, Plasma-Diagnostics. Ed. North-Holland, Amsterdam (1968)

[6] V. P. Gavrilenko, H. J. Kim, T. Ikutake, J. B. Kim, Y. W. Choi, M. D. Bowden, K. Muraoka. "Measurement method for electric fields based on Stark spectroscopy of argon atoms”. Phys. Rev. E, 62, 7201. (2000)

[7] D. Spasojević, V. Steflekova, N. M. Šišović, N. Konjević. "Electric field distribution in the cathode-fall region of an abnormal glow discharge in hydrogen: experiment and theory”. Plasma Sources Sci. Technol., 21025006 (2012).

[8] G. S. He, S. H. Liu, Physics of Nonlinear Optics. World Scientific (1999)

[9] K. Liu, M. G. Littman, "Novel geometry for single-mode scanning of tunable lasers", Opt. Lett., 6, 117118 (1981)

[10] B. Barbieri, N. Beverini, A. Sasso, “Optogalvanic spectroscopy”, Rev. Mod. Phys., 62, 603 (1990)

[11] C. Pérez, M. I. de la Rosa, K. Grützmacher. "Hollow cathode fall field strength measured by Doppler-free two-photon optogalvanic spectroscopy via Stark splitting of the $2 S$ level of hydrogen", Eur. Phys. J. D, 56, 369-375 (2010)

[12] M. Garcia-Lechuga, L. M. Fuentes, K. Grützmacher, C. Pérez, and M. I. de la Rosa, "Calculation of the spatial resolution in two-photon absorption spectroscopy applied to plasma diagnosis", J. Appl. Phys., 116, 133103 (2014)

[13] V. Gonzalez-Fernandez, K. Grützmacher, C. Pérez and M. I. de la Rosa, "Experimental study of the electric field in a hollow cathode discharge in hydrogen: influence of sputtering" J. Instrum., 12, C11029 (2017)

[14] V. Gonzalez-Fernandez, K. Grützmacher, A. Steiger, C. Pérez and M. I. de la Rosa, "Measured cathode fall characteristics depending on the diameter of a hydrogen hollow cathode discharge" Plasma Sources Sci. Technol., 26, 105004 (2017)

[15] V. Gonzalez-Fernandez, K. Grützmacher, C. Pérez and M. I. de la Rosa, "Influence of the cathode material in the cathode fall characteristics of a hydrogen hollow cathode glow-discharge" J. Appl. Phys., 124, 033302 (2018)

\section{Introduction}

Plasma science is nowadays a very active field of work due to its immense potential in basic research and a huge range of applications, from medicine and surgery to industrial implementation, and of course fusion science. Working with plasmas is not always easy; therefore, it is crucial to know as much information as possible about the plasma. Knowing the plasma state requires determining all the parameters that mark its behaviour. Most of the plasmas of interest have complex spatial distributions outside the thermodynamic equilibrium. For this reason, the development of techniques allowing independent measurement of the different parameters characterizing the discharge is fundamental for its correct diagnosis.

Optical techniques have always been a powerful ally to study plasmas because they provide non-intrusive diagnostic [1]. Following an historical line, optical emission is the first method employed, and during decades it was almost the only one available. But this trend changed with the apparition of lasers. The monochromatic light of lasers opened a whole new way to interact with plasmas: for the first time there was the possibility of active access to plasmas, through their atomic and molecular transitions [2]. This possibility also implied an exciting technological challenge: to be able to induce transitions it is necessary to have radiation with the exact wavelength and very narrow spectrally. In these terms, non-linear optics, and some of its associated processes (frequency addition and subtraction, parametric amplification, etc.) is an extremely powerful tool for generating ad-hoc radiation for different applications [3].

In the $\mathrm{PhD}$ thesis that this article resumes [4], a high-resolution laser spectroscopy diagnostic was implemented to study a hydrogen hollow-cathode discharge, operated in glow-discharge regime. The study is done via the experimental determination of the electric field (E-field) in the cathode fall region, i.e. the nearest region to the cathode surface. The electric field strength value is a very important parameter because it controls the particle flux and their energy distribution [5]. It depends on several factors, as the 
discharge geometry, the cathode material, pressure, current, etc. The cathode region uses to be narrow in this type of devices, therefore a high-spatial resolution method is required. Different authors have developed different techniques for the measurement of the electric field in different types of discharges with accurate results (see for instance [6,7]). In our case, some of these techniques were not suitable, because our object of interest, i.e. the cathode region, is about only $2 \mathrm{~mm}$ length. Therefore, techniques as emission spectroscopy could not gave us the necessary features in terms of spatial resolution.

The technique employed in this thesis is based in the Stark shifting and splitting of the $2 \mathrm{~S}$ level of atomic hydrogen, reached by the simultaneous absorption of two $243 \mathrm{~nm}$ photons, circularly polarized in opposed senses and in counter-propagating beams to avoid Doppler effect. The E-field value is determined comparing the splitting of the Stark components with the theoretical values. In the following sections, the whole experimental device will be fully explained, as well as the method developed to measure the electric field and a brief resume of the different measurement campaigns and the main results obtained.

\section{Experimental arrangement}

In this section the whole experimental set-up is carefully explained, paying special attention to the laser system and the optical path designed to measure the E-field.

\section{2.a. Laser spectrometer}

The laser system is composed of two parts: first a $10 \mathrm{~Hz}$ injection-seeded Q-switched Nd:YAG laser (Continuum, Powerlite 9000). This laser is able to generate the second and third harmonic of the fundamental wavelength, 532 and $335 \mathrm{~nm}$, following respectively non-linear and parametric processes of second harmonic generation (SHG) and third harmonic generation via sum-frequency generation [3]. These non-linear processes require high irradiancies, to give an approximate idea of the laser power it must be taken into account that only the $10 \%$ of the SHG is extracted from the laser ( $18 \mathrm{~mJ}$ ) while the rest goes to the generation of the third harmonic, giving finally $100 \mathrm{~mJ}$ of $355 \mathrm{~nm}$ radiation.

Both wavelengths are introduced in a laser spectrometer (Mirage 500, Continuum), who will generate the $243 \mathrm{~nm}$ UV radiation required for spectroscopic measurements. For this experiment the final wavelength is fixed at $243 \mathrm{~nm}$, but its tuning possibilities would also allow the generation different radiations in the ultraviolet range for the study of other elements such as oxygen $(225.8 \mathrm{~nm})$, nitrogen $(211 \mathrm{~nm})$ and carbon $(280.4 \mathrm{~nm})$ through two-photons transitions. All the non-linear processes to get the $243 \mathrm{~nm}$ wavelength are clearly indicated in Figure 1, also showing also the complementary wavelengths obtained in the different processes [8].

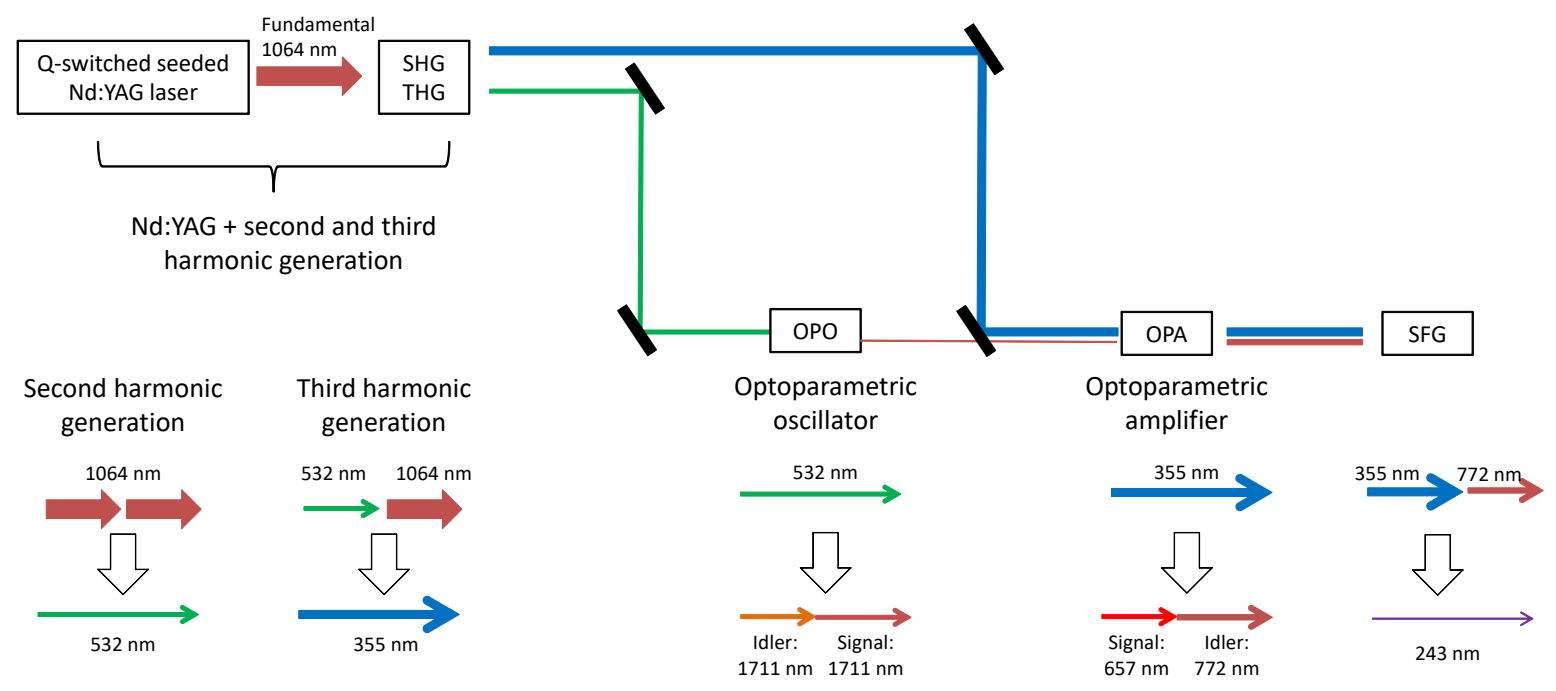

Figure 1: Scheme of all the non-linear processes in the laser Mirage 500

The first step in this laser is an Optical-Parametric Oscillator (OPO), the scheme can be seen in Figure 2 . An OPO is an optical cavity, in this case, following the configuration given in [9]. The $532 \mathrm{~nm}$ radiation 
(second harmonic of the Nd:YAG) is introduced into the cavity and reflected in a dichroic mirror, falling upon the diffraction grating. The KTP crystal at the centre of the cavity suffers a non-linear process (difference of frequencies), emitting two new radiations: $772 \mathrm{~nm}$ and $1711 \mathrm{~nm}$ [3]. After being reflected in the rear mirror, the output radiation returns to the diffraction grating, where due to the configuration of the cavity, the first order of diffraction of the most energetic radiation is normally reflected in the tuning mirror, so it returns along the same path, coupling to the cavity. In this way, the return radiation acts as a seed in the process. All control elements ensure that the output radiation has a single longitudinal mode, compulsory condition for carrying out spectroscopic measurements.

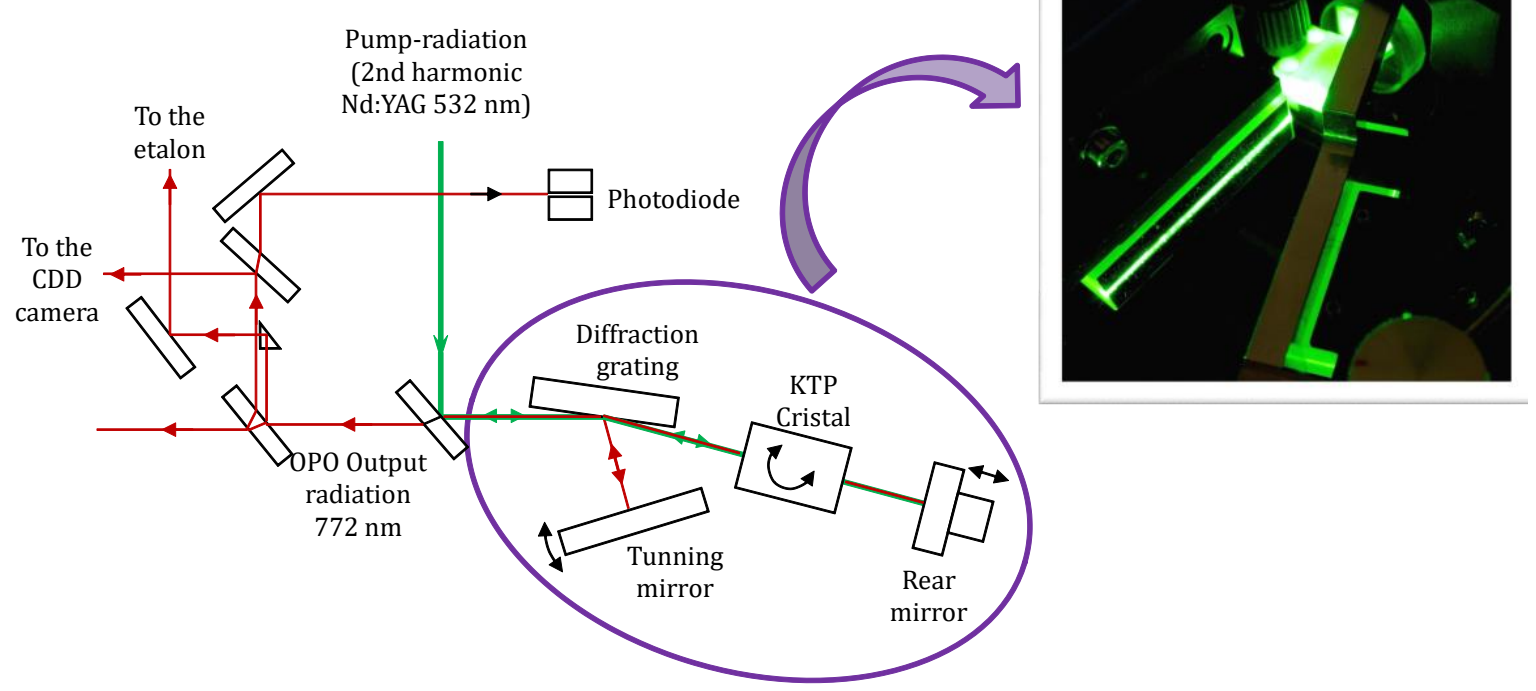

Figure 2. Scheme of the OPO cavity.

The possibility that the radiation from the OPO can be tuned lies in the ability to rotate the KTP glass in a horizontal plane, so that the angle of incidence of the second harmonic with respect to the optical axis of the glass varies. Practically, that means that the new pair of generated wavelengths changes with the crystal tuning. In conjunction with the movement of the glass, the tuning mirror moves to redirect the first order of the signal into the cavity. This movement modifies the length of the cavity, so the rear mirror (at normal incidence) is continuously coupled to maintain a single longitudinal mode.

After the OPO cavity, the $772 \mathrm{~nm}$ radiation needs to be amplified. Two Optical Parametric Amplifiers (OPA-BBO crystals) are pumped with the third harmonic of the ND:YAG (335 nm). In this case, the nonlinear process is the difference of frequencies, giving as a result $657 \mathrm{~nm}$, and in addition the $772 \mathrm{~nm}$ wavelength is amplified. The $657 \mathrm{~nm}$ is used for alignment and diagnostics.

The last step is a sum frequency generation (SFG) where the remaining $355 \mathrm{~nm}$ and the amplified $772 \mathrm{~nm}$ are introduced together in a BBO crystal, finally obtaining the $243 \mathrm{~nm}$ wavelength. This laser spectrometer provides up to $10 \mathrm{~mJ}$ pulse energy in $2.5 \mathrm{~ns}$ and $300 \mathrm{MHz}$ bandwidth (Fourier transform limit) in this configuration. An etalon with a free spectral range of about $7 \mathrm{GHz}$ and a photodiode (PD) are used to control the regular tuning of the laser frequency by monitoring the OPO signal $(772 \mathrm{~nm})$.

\section{2.b. Optical design}

A scheme of the whole optical design can be seen in Figure 3. The radiation is divided into two beams with an identical optical path, so that a pulse and its twin are in the centre of the discharge source. In one of the two paths a retro-reflector has been installed, which allows the path of the beam to be modified and compensates for a slight movement in its trajectory, i.e. if one of the two beams moves horizontally, the other will move in the same direction. 


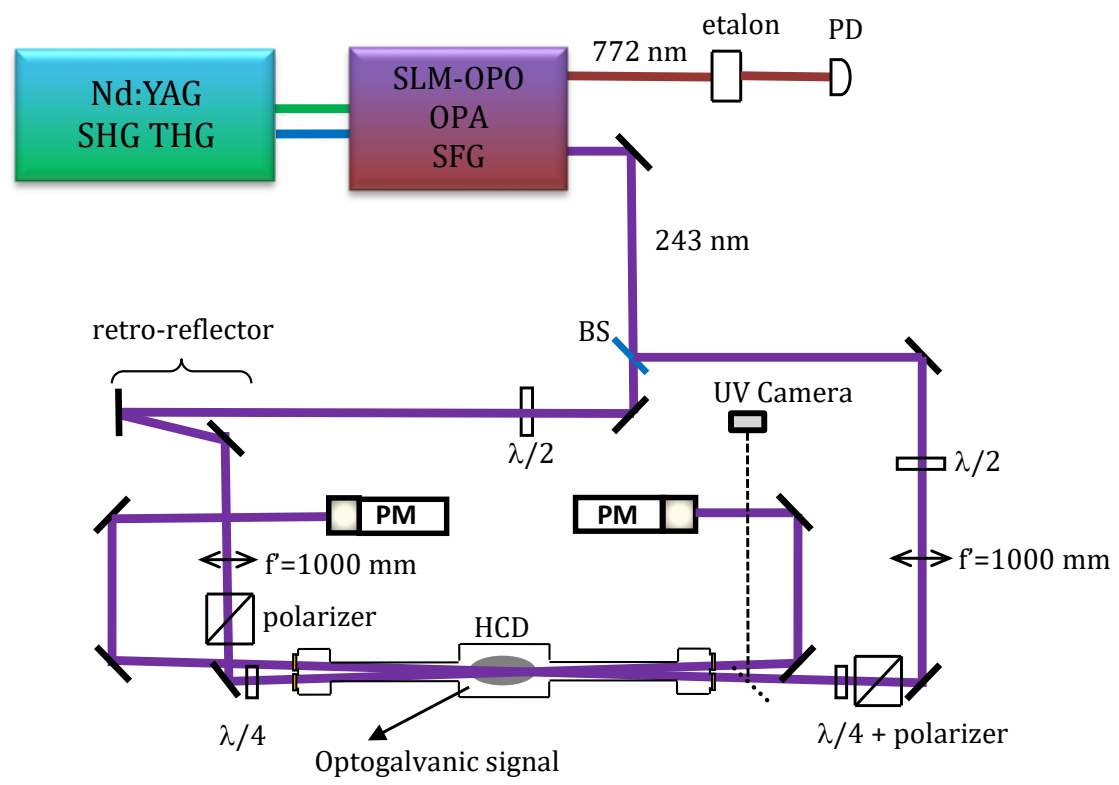

Figure 3. Scheme of the experimental arrangement. PD: photo-diode; PM:photo-multiplier, BS : beam splitter; HCD: hollow cathode discharge.

Each of the beams is circularly polarized by means of a linear polarizer and a zero-order $\lambda / 4$ waveplate. The beams travel in opposite directions to obtain Doppler-free measurements and are overlapped at the top of the discharge lamp by lenses with a focal length of one meter. The overlapping is carried out with an angle of $1.53^{\circ}$, the minimum possible to allow both beams to enter the discharge source, and to ensure the smallest possible overlapping volume, of about $100 \mu \mathrm{m}$ in diameter and $100 \mathrm{~mm}$ in length. This subDoppler resolution is strictly necessary to achieve high resolution measurements, as can be seen in Figure 1Figure 4. If $\mathrm{t}$ both beams are not counter-propagating, a much larger signal is obtained, masking the splitting of the $2 \mathrm{~S}$ level of hydrogen or deuterium. The signal increase is consequence of the there is no longer overlapping, and $1 \mathrm{~S}-2 \mathrm{~S}$ transition and subsequent photo-ionization is produced throughout the whole discharge.

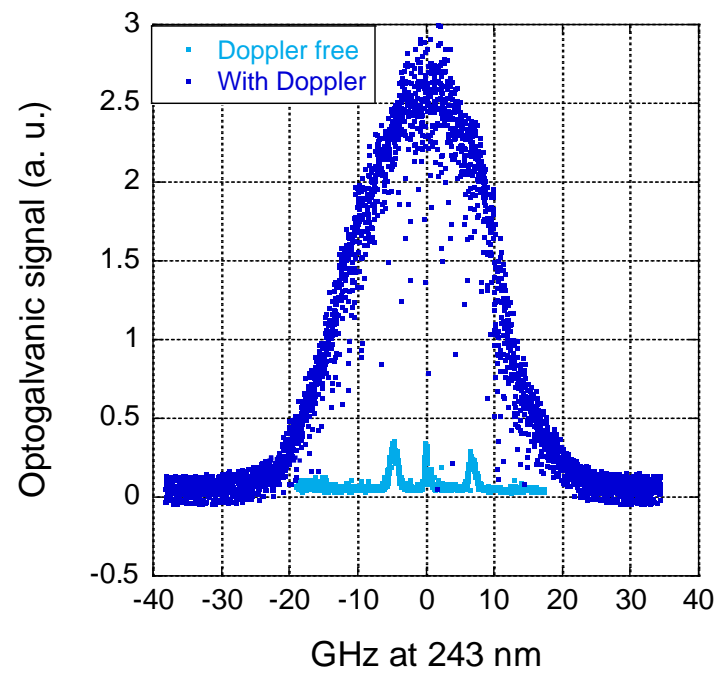

Figure 4: Comparison of the spectra acquired following Doppler-free configuration (light blue) and with Doppler effect (dark blue). The increasing od the signal in the Doppler case is due to there is no limit in the overlapping and both beams share all the way inside the plasma source, inducing more photo-ionization. Measurements recorded in a hydrogen discharge, with a $10 \mathrm{~mm}$ inner diameter tungsten cathode, with $400 \mathrm{~Pa}$ of pressure, $100 \mathrm{~mA}$ of current at $100 \mu \mathrm{m}$ from the cathode surface.

Beam intensities are controlled with a $\lambda / 2$ waveplate followed by a linear polarizer; these two elements constitute a variable attenuator. To check that the energy is constant during all the measurement process, 
two photomultipliers (Hamamatsu, series R5600U-03, PM in Figure 3). These photomultipliers need to be polarised at a voltage of $800 \mathrm{~V}$ for correct operation.

\section{2.c. Hollow cathode discharge}

The plasma source is formed by an external structure of brass with three bodies cylindrically symmetrical, separated by Teflon discs to isolate them electrically. The central piece is the cathode (50 mm length), and the two outer pieces are the anodes. The separation between the cathode and both anodes is about $10 \mathrm{~mm}$. All the pieces have an axial perforation, allowing the performance of end-on spectroscopic measurements. This configuration allows the insertion of cathodes of different diameter and material. This configuration has been proven to generate a symmetrical discharge and to provide greater stability to the discharge, thus significantly reducing the noise recorded in the detection. The gas (hydrogen or deuterium) enters through one anode and exits through the other, at a constant flow rate of approximately $10 \mathrm{~cm}^{3} \mathrm{~s}^{-1}$. The anodes and cathodes are independently water-cooled. An scheme and a photograph of the discharge source is shown in Figure 5, where the luminosity of the hydrogen plasma can be seen through the Teflon discs. The lamp is mounted on two micrometric displacers that allow movement in both horizontal and vertical directions, making alignment precise and simple. The vertical translator also allows measurements at different radial distances from the cathode surface, d. The geometry of the experiment and the fact that the laser beams are not punctual, establishes a minimum distance of $160 \mu \mathrm{m}$ from the cathode surface. The resonant two-photon excitation and subsequent photoionization (absorbing a third photon, never mind the beam) causes the optogalvanic signal [10]. This photo-ionization increases the electron density in the measurement volume, and it is detected as a transient voltage drop between the cathode and one of the anodes. The optogalvanic signal is recorded by a four-channel oscilloscope (Keysight Technologies Inc. model DSOS104A) synchronized with the $10 \mathrm{~Hz}$ rate repetition of the $\mathrm{Nd}$ :YAG laser.
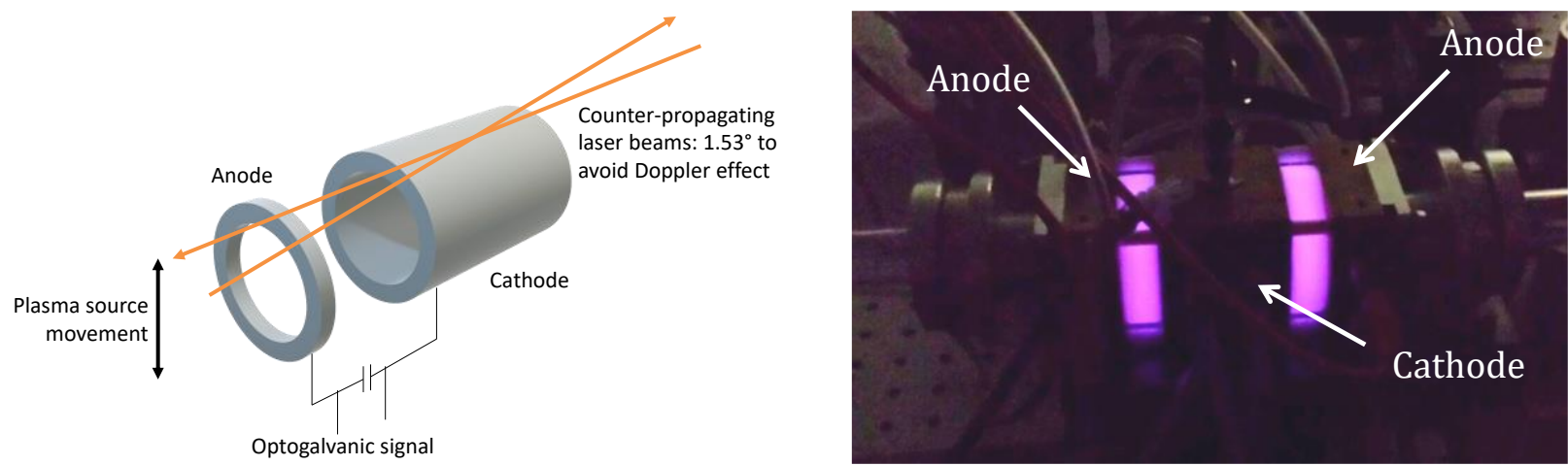

Figure 5: Left: Scheme of the overlapping of the two laser beams, they are focalized in the upper-central part of the hollow cathode. The peaked anodes are represented as rings for a better visualization. Right: Photograph of the discharge source, with the two anodes and the cathode, and the characteristics luminosity of a hydrogen plasma through the isolating Teflon discs.

\section{Electric field determination}

As it was already mentioned, the E-field determination is done via the Stark shifting and splitting of the $2 \mathrm{~S}$ level of hydrogen in three different components, $2 \mathrm{P}^{1 / 2}, 2 \mathrm{~S}^{1 / 2}$ and the $2 \mathrm{P}^{3 / 2}$. The separation in $\mathrm{GHz}$ of the extreme components $\left(2 \mathrm{P}^{1 / 2}\right.$ and the $\left.2 \mathrm{P}^{3 / 2}\right)$, so $\left.\Delta v=v\left(2 \mathrm{P}^{1 / 2}\right)-v\left(2 \mathrm{P}^{3 / 2}\right)\right)$, compared with theoretical calculations [11] determine the local $E$-field strength value. This process can be seen in Figure 6. 
Typical spectrum

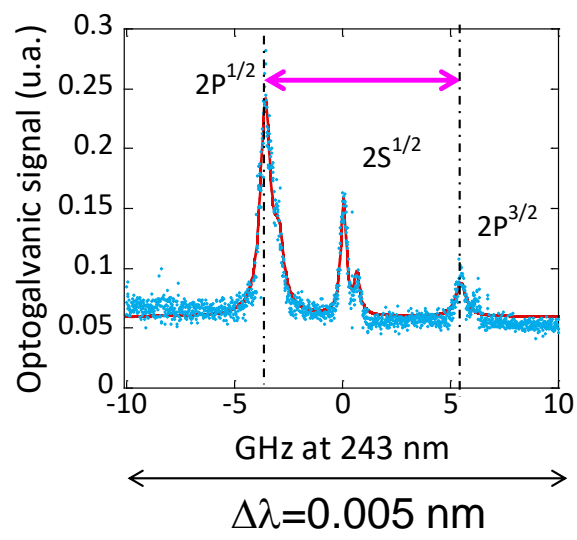

Stark splitting of the $2 \mathrm{~S}$ level of Hydrogen

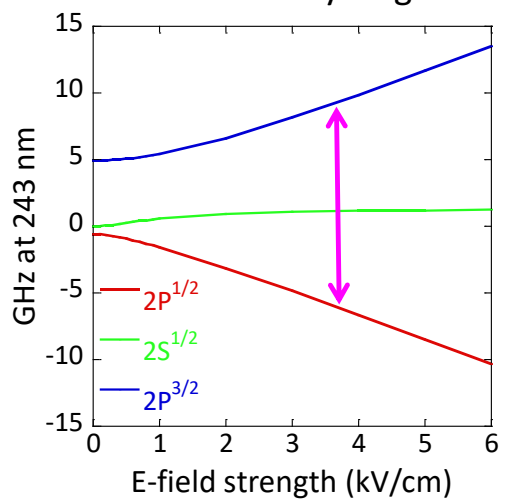

Figure 6: Schematic representation of the relationship between the frequency separation of the different Stark components of the spectrum and their relation to the value of the associated local electric field.

Of course, the E-field determination can be done with three frequency differences available: $2 \mathrm{P}^{1 / 2}-2 \mathrm{P}^{3 / 2}$, $2 \mathrm{P}^{1 / 2}-2 \mathrm{~S}^{1 / 2}$ and $2 \mathrm{~S}^{1 / 2}-2 \mathrm{P}^{3 / 2}$. The maximum difference between them was found to be $5 \%$, due to the laser tuning. However, for the final values, the $2 \mathrm{P}^{1 / 2}-2 \mathrm{P}^{3 / 2}$ difference was chosen, because by covering a wider range in $\mathrm{GHz}$ can compensate for small deviations in the laser tuning. The detection limit of this technique is determined by the decrease of signal that is suffered when we move away from the cathode surface, as can be seen in Figure 7.

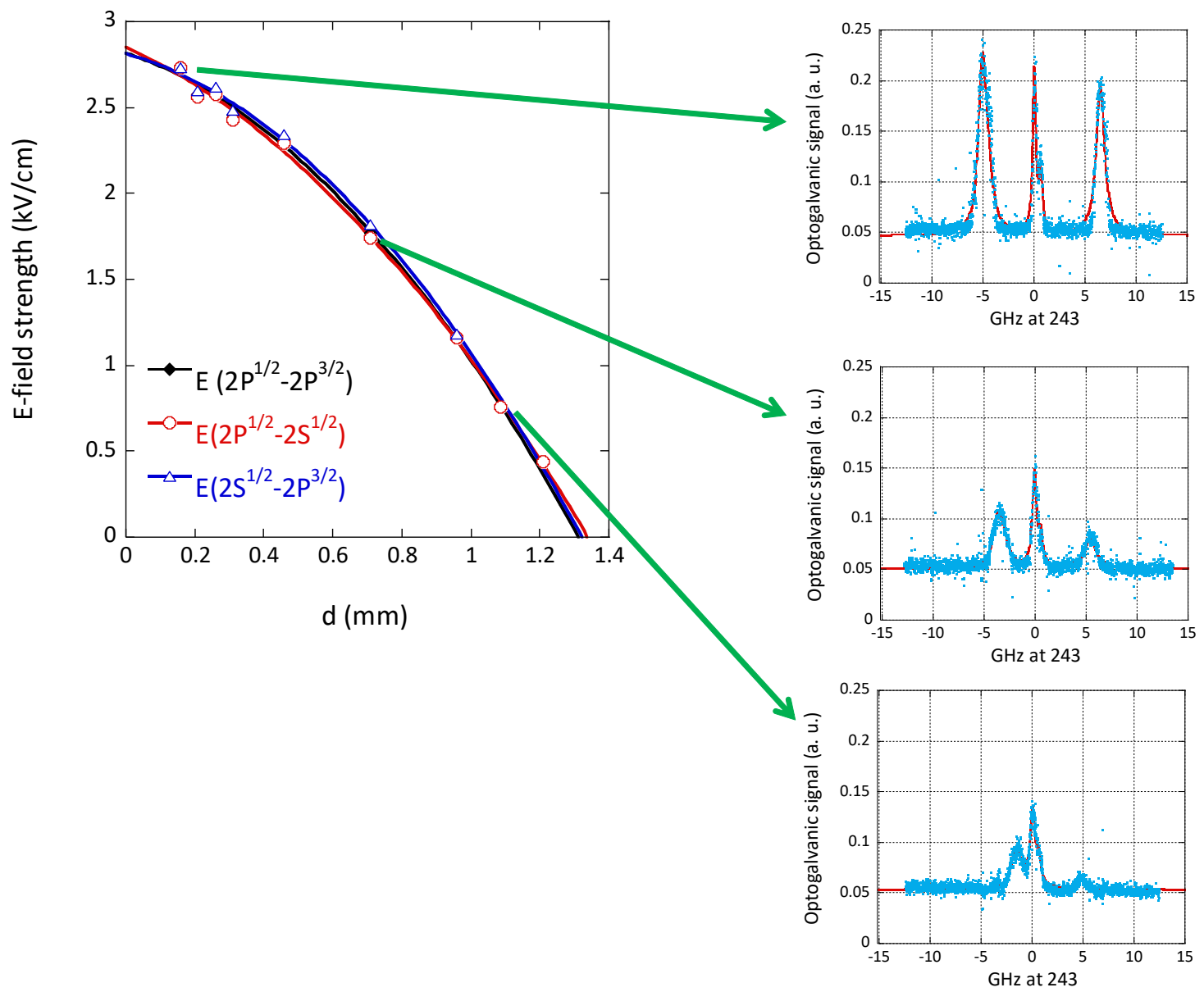

Figure 7: Electric field determined by the three possible frequency differences: $2 \mathrm{P}^{1 / 2}-2 \mathrm{P}^{3 / 2}, 2 \mathrm{P}^{1 / 2-2}-2 \mathrm{~S}^{1 / 2}$ and $2 \mathrm{~S}^{1 / 2}-2 \mathrm{P}^{3 / 2}$ in a hydrogen discharge, steel cathode $15 \mathrm{~mm}$ diameter stainless steel, a pressure of $600 \mathrm{~Pa}$ and a current of $225 \mathrm{~mA}$. 
Each E-field value has associated an uncertainty value based on the small lack of regularity of the laser frequency tunning. An etalon of $7 \mathrm{GHz}$ of free spectral range is responsible of controlling the laser tunning. The standard deviation of the fringe separation is $0.16 \mathrm{GHz}$ for a usual set of measurements, half the bandwidth of the laser. Nevertheless, for each plasma condition, particular errors are allotted to each measurement.

\section{Results of the different measurement campaigns}

In this section the main measurements campaigns are summarized as well as the main results. The measurements were performed in four different cathodes, two made of tungsten and two made of stainless steel; with different inner diameters, 10 and $15 \mathrm{~mm}$. Measurements were carried out in a wide range of experimental conditions, with currents ranging from 50 to $300 \mathrm{~mA}$ and pressures between 400 and $1350 \mathrm{~Pa}$.

\section{4.a. Influence of the laser power}

As explained above, both spatial and spectral resolution is crucial in this experiment. The spatial resolution of the experiment was determined via a UV camera (recording foci images) and subsequent calculus. The result was that optogalvanic detection can provide a high-spatial resolution since up to $150 \mathrm{MW} / \mathrm{cm}^{2}$ laser irradiance. In these conditions more than $75 \%$ of the signal is created in a small central area of the overlap volume of only $100 \mu \mathrm{m} \times 100 \mu \mathrm{m}$ and a length of $10 \mathrm{~mm}$ [12].

Before the two main measurement campaigns, some test measurements were made with high and low energy, to check that limit of $150 \mathrm{MW} / \mathrm{cm}^{2}$ of irradiance in the overlapping volume. The measurements were made on two $10 \mathrm{~mm}$ diameter cathodes, one of stainless steel and one of tungsten. In the tungsten cathode two measures were conducted with low energy $(45 \mu \mathrm{J})$ and high laser energy with $600 \mathrm{~Pa}$ pressure and $100 \mathrm{~mA}$ current. The two spectra lead to same E-field value $( \pm 3 \%)$. A little difference is caused by a slight saturation: the fundamental level becomes empty, and the photo-ionization reduces the lifetime of the $2 \mathrm{~S}$ level. However, in stainless steel the difference is much higher (see Figure 8) where a discharge of $900 \mathrm{~Pa}$ and $100 \mathrm{~mA}$ is shown. Low energy spectrum was recorded with low energy $(45 \mu \mathrm{J})$, and pink one was recorded with a laser energy of $225 \mu \mathrm{J}$. The first thing to notice is how the is how the $2 \mathrm{P}^{1 / 2}$ and $2 \mathrm{P}^{3 / 2}$ components of the high-energy measured spectrum are much more separated, which leads to a higher value of the electric field, which grows from $2.3 \mathrm{kV} / \mathrm{cm}$ up to $2.83 \mathrm{kV} / \mathrm{cm}$. This growth is too large to be attributed to any uncertainty, nor can it be related neither to saturation effects due to level depletion nor to the reduction of the average life of the $2 \mathrm{~S}$ level. Any of these effects would cause such only a widening of the Stark components, and a moderate growth of the electric field, due to the Stark effect. Therefore, it can be concluded that the energy per pulse is critical in determining the electric field. Especially when stainless steel is used as cathode material, not only is the spatial resolution lost in the spectra, but the value of the electric field in the entire cathode area is overestimated due to the additional displacement of the components. The whole study is presented in [13].

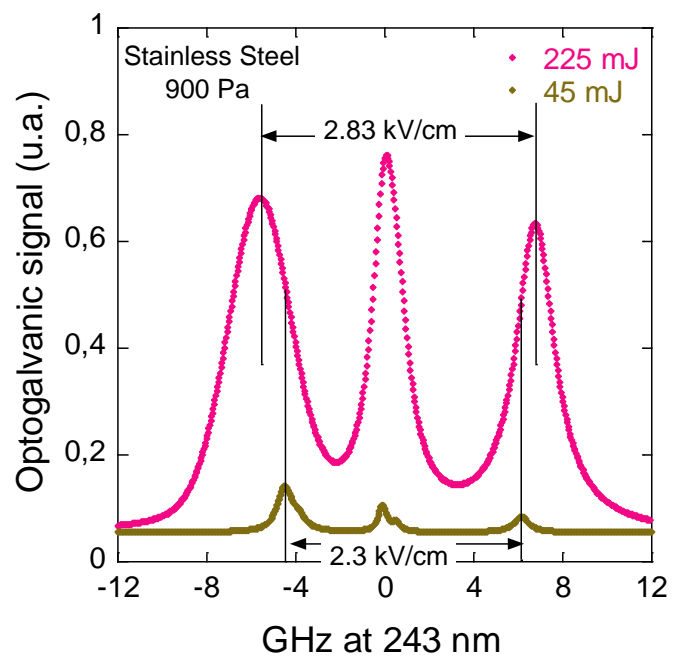

Figure 8: Comparison of two spectra measured at $160 \mu \mathrm{m}$ from the surface of a $10 \mathrm{~mm}$ diameter Stainless steel cathode, 900 Pa and $100 \mathrm{~mA}$. Rose line indicates high laser energy (LE), brown lines low laser energy. 


\section{4.b. Influence of the cathode diameter on the electric field strength fall}

The first of the studies consisted of studying how the geometry of the discharge affected the fall of the electric field. The study was conducted in the two tungsten cathodes, keeping always the same current density. To maintain the same current density in cathodes of different diameters, different current values must be imposed. For example, if we want a density of $6.37 \mathrm{~mA} / \mathrm{cm}^{2}$, in a $10 \mathrm{~mm}$ cathode the current should be $100 \mathrm{~mA}$, while in a $15 \mathrm{~mm}$ cathode, the imposed current will be $150 \mathrm{~mA}$. Figure 9 show a representative graph comparing two different measurements in the two cathodes, with the same current density to compare them properly:

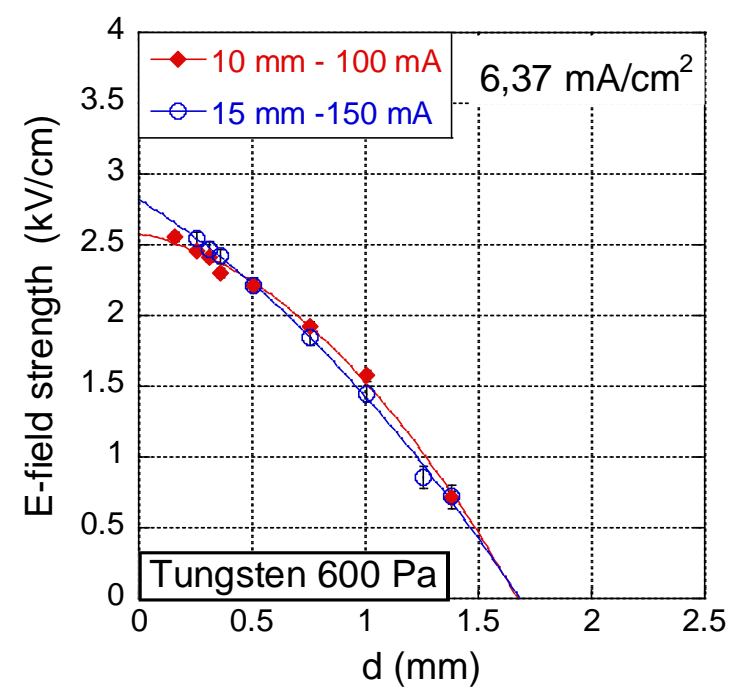

Figure 9: Electric field strength vs. the distance from the cathode surface, for 10 and $15 \mathrm{~mm}$ inside diameter tungsten cathodes. The discharges were operated in 600 Pa pressure. Red diamonds correspond to $10 \mathrm{~mm}$ cathode and blue circles to $15 \mathrm{~mm}$ cathode. Both current values are elected to keep the same current density in both cases.

As can be clearly seen, while keeping the same current density, the E-field strength in both cathode diameters is practically the same. The study was repeated in different pressures (400 and $900 \mathrm{~Pa}$ ) and also in different currents (from 50 to $300 \mathrm{~mA}$ ), leading to the same conclusion: there is no significant difference in the E-field strength due to the cathode diameter when the current density is the same for both diameters. This fact allows that the experimental data obtained in this thesis of the measurement of the electric field can be compared with the data of discharges of very different geometries, with those discharges of parallel plane electrodes. Therefore, our data are valid to test one dimensional modelling of the cathode fall region of low-pressure hydrogen discharge. The whole study can be consulted in [14]. 


\section{4.c. Influence of the cathode material: sputtering}

Having verified that the diameter of the discharge was not a differentiating parameter when measuring the fall of the electric field, it was the turn of the cathode material used. For this, we used $15 \mathrm{~mm}$ inner diameter cathodes (which have a wider cathode area). Both materials have been widely used in this kind of discharges; there lies the interest in knowing which material is better for generating pure hydrogen plasmas. In Figure 10, two E-field strengths measured with $600 \mathrm{~Pa}$ and $300 \mathrm{~mA}$ current in the two different materials:

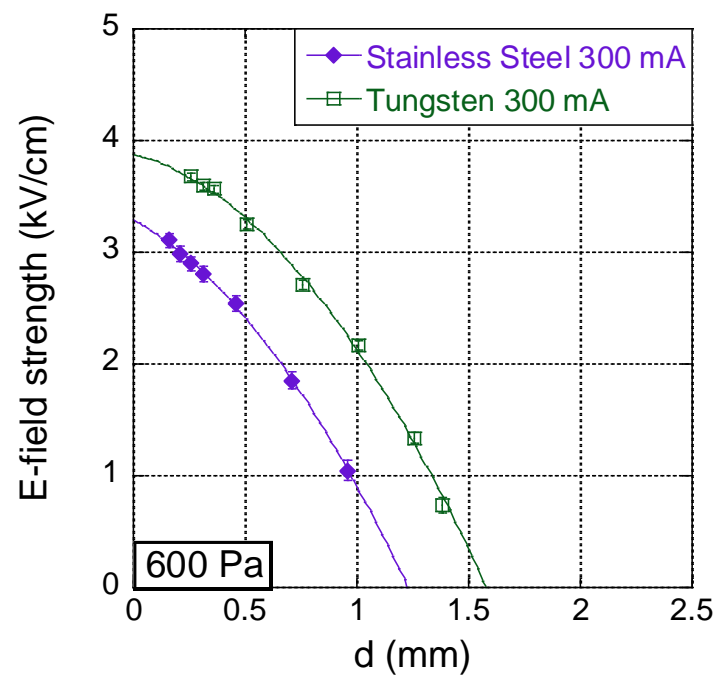

Figure 10: Electric field strength vs. the distance from the cathode surface, for $15 \mathrm{~mm}$ inside diameter tungsten and stainless-steel cathodes. The discharges were operated in $600 \mathrm{~Pa}$ pressure and $300 \mathrm{~mA}$ current. Purple diamonds correspond to stainless steel cathode and green squares to tungsten mm cathode.

In this study, that can be fully read in [15], the measurements showed that the E-field strength values are always higher in tungsten that in stainless steel. The reason is that sputtering (metallic particles emitted from the cathode surface) rate was much higher in stainless steel than in tungsten. Sputtered material increases the density of charged particles; therefore it increases the plasma conductivity. That leads to a lower E-field strength values in stainless steels when driving the same current value [13]. This result proves that the plasma generated in tungsten serve as pure hydrogen plasma, being the sputtering rate negligible in most discharge conditions.

\section{4.d. Perspective: measurements in Deuterium}

Finally, and as future perspectives of this work, it is indicated that this experiment is easily adaptable for the performance of E-fields measurements in the same discharge but operated in deuterium as buffer gas. The interest of this study lies in the fact that both isotopes are practically identical, deuterium having a mass twice that of the hydrogen atom. Therefore, the comparison of electric field measurements in both isotopes would be extremely interesting, which opens a second new line of research. Due to the mass difference between hydrogen and deuterium, the output wavelength of the laser must be slightly modified from the required value for hydrogen measurements, with a difference of about $0.03 \%$.

\section{Summary}

This article shows a brief summary of the experimental arrangement, the measure procedure and the main results of my $\mathrm{PhD}$ thesis, putting special emphasis on the entire laser device used for the diagnosis of hydrogen plasmas. Laser spectroscopy provides a high-resolution solution to properly measure the electric field present in a hollow cathode discharge. The E-field is determined via the splitting and shifting of the $2 \mathrm{~S}$ level of hydrogen, due to the Stark effect. The hydrogen atoms suffer two-photon absorption provided by two counter-propagating circularly polarized beams of opposite directions, to avoid Doppler effect. A third photon is absorbed, inducing a tiny change in the plasma impedance, usually called optogalvanic detection. The E-field is determined by measuring the frequency shift between the $2 \mathrm{P}^{1 / 2}$ and 
the $2 \mathrm{P}^{3 / 2}$ components. A complex UV laser spectrometer, based on non-linear crystals, provides highpower single-longitudinal mode and tuneable $243 \mathrm{~nm}$ radiation with $2.5 \mathrm{~ns}$ temporal duration and 300 $\mathrm{MHz}$ bandwidth. The E-field measurements were performed for pressures varying from $400 \mathrm{~Pa}$ to $1350 \mathrm{~Pa}$ and for currents from 50 to $300 \mathrm{~mA}$. Measurements were conducted with four different cathodes: two made of tungsten and two made of stainless steel, with two different diameters: 10 and $15 \mathrm{~mm}$. The measurement campaigns revealed three main results: (a) the measurements must be done with a moderate irradiance $(45 \mu \mathrm{J})$ in order to avoid saturation in the spectra and the subsequent loss of resolution; (b) there is no significant difference between the E-field strength values in cathodes of different diameter when keeping the same current density and (c) the sputtering rate of stainless steel is much higher than tungsten, and only with tungsten cathodes it is possible to ensure pure hydrogen plasmas.

\section{Acknowledgements}

The author thanks DGICYT (Ministerio de Economía y Competitividad) for the project ENE2012-35902, FEDER funds and the grants BES-2013-063248 given to V. González-Fernández. The author thanks M. I. de la Rosa, C. Pérez and A. Steiger for being the supervisors of this thesis. The author thanks also the invaluable help of K. Grützmacher, we miss you so much. 